\title{
Systematic review of the prevalence of current smoking among hospitalized COVID-19 patients in China: could nicotine be a therapeutic option? Reply
}

\author{
Konstantinos Farsalinos ${ }^{1,2}(\mathbb{D}) \cdot$ Anastasia Barbouni $^{2} \cdot$ Raymond Niaura $^{3}$
}

Received: 17 July 2020 / Accepted: 20 July 2020 / Published online: 21 September 2020

(c) Società Italiana di Medicina Interna (SIMI) 2020

Dear editor,

We would like to thank Dr. Takagi [1] for his comments and the interesting analysis of the association between COVID19 incidence and smoking prevalence in Japanese prefectures. In this response, we clarify some possible misunderstandings related to the current research on the link between smoking and COVID-19.

Our systematic review and meta-analysis examined the prevalence of smoking among hospitalized COVID-19 patients only [2]. Our findings cannot be interpreted as smoking reducing the risk of being infected with SARSCoV-2 and developing COVID-19. In fact, we emphasized that no conclusion can be drawn about the prevalence of current smoking among patients with less severe COVID19 that would not require hospitalization. While we suggested that nicotine is unlikely to have direct antiviral activity and, thus, is not expected to act as chemoprophylaxis, recent studies suggest that smokers may be less likely to be diagnosed with COVID-19 [3], while an inverse association between national smoking rates and COVID-19 mortality was reported [4]. These findings may not be in agreement with the report by Dr. Takagi but, in any case, the overall incidence of COVID-19 (including mild forms of the disease) should be clearly differentiated from severe

The article belongs to COVID 19.

Konstantinos Farsalinos

kfarsalinos@gmail.com

Department of Pharmacy, University of Patras, Rio, Greece

2 School of Public Health, Department of Public and Community Health, University of West Attica, Athens, Greece

3 Departments of Social and Behavioral Science and Epidemiology, College of Global Public Health, New York University, New York City, USA
COVID-19 that requires hospitalization. Additionally, the lower than expected prevalence of smoking among hospitalized COVID-19 patients is not contradictory to the reported higher odds for adverse outcome in hospitalized smokers compared to non-smokers. In our most recent systematic review and meta-analysis of 30 studies, we reported both an unexpectedly low prevalence of smoking among hospitalized COVID-19 patients and higher odds for adverse outcome in hospitalized smokers [5]. In reality, the evidence suggests that any association between smoking and disease outcome cannot not be generalized to the whole population of smokers but should refer specifically to the seemingly low proportion of smokers who develop severe COVID-19 necessitating hospitalization. One potential explanation for the adverse outcome among hospitalized smokers is that they experience abrupt nicotine cessation from the moment they are admitted to the hospital (unless they are given nicotine replacement therapies). As a result, these patients will be deprived of any potentially beneficial effects of nicotine, which may be related to the activation of the cholinergic anti-inflammatory pathway and/or the prevention of a direct interaction between SARS-CoV-2 and nicotinic acetylcholine receptors, as we have presented in our hypothesis [2, 5]. Concerning the effects of smoking on ACE2 expression, there is highly conflicting and contradictory evidence $[2,5]$. Even if smoking was found to up-regulate ACE2, this does not necessarily imply adverse prognosis. In fact, ACE2 has strong anti-inflammatory, anti-oxidant and antifibrotic properties, and it is possible that ACE2 upregulation is protective against severe COVID-19 despite being used by SARS-CoV-2 as a receptor for cell entry [5]. Finally, we take this opportunity to emphasize once again that smoking cannot be considered a protective factor for COVID-19, and smokers should still be encouraged to quit. However, the potential therapeutic effects of nicotine or other nicotinic cholinergic agonists should be further explored in in vitro studies and clinical trials. 


\section{Compliance with ethical standards}

Conflicts of interest KF reports no competing interests for the past 36 months. For the past 60 months, one study was funded by the nonprofit association Tennessee Smoke-Free Association. AB reports no conflict of interest. RN receives funding from the Food and Drug Administration Center for Tobacco Products via contractual mechanisms with Westat and the National Institutes of Health. Within the past three years, he has served as a paid consultant to the Government of Canada and the FDA via contracts with Industrial Economics Inc., and has received an honorarium for a virtual meeting from Pfizer Inc.

Ethical approval Not applicable.

Informed consent Not applicable.

\section{References}

1. Takagi H (2020) Systematic review of the prevalence of current smoking among hospitalized COVID-19 patients in China: could nicotine be a therapeutic option? Comment. Intern Emerg Med. https://doi.org/10.1007/s11739-020-02396-y
2. Farsalinos K, Barbouni A, Niaura R (2020) Systematic review of the prevalence of current smoking among hospitalized COVID-19 patients in China: could nicotine be a therapeutic option? Intern Emerg Med 9:1-8. https://doi.org/10.1007/s11739-020-02355-7

3. Israel A, Feldhamer I, Lahad A, Levin-Zamir D, Lavie G (2020) Smoking and the risk of COVID-19 in a large observational population study. medRxiv. https://doi.org/10.1101/2020.06.01.20118 877

4. Norden MJ, Avery DH, Norden JG, Haynor DR (2020) National smoking rates correlate inversely with COVID-19 mortality. medRxiv. https://doi.org/10.1101/2020.06.12.20129825

5. Farsalinos K, Barbouni A, Poulas K, Polosa R, Caponnetto P, Niaura R (2020) Current smoking, former smoking, and adverse outcome among hospitalized COVID-19 patients: a systematic review and meta-analysis. Ther Adv Chronic Dis 11:2040622320935765. https://doi.org/10.1177/2040622320 935765

Publisher's Note Springer Nature remains neutral with regard to jurisdictional claims in published maps and institutional affiliations. 